\title{
An Air Indexing Method for Encrypted XML Data Broadcast in Mobile Wireless Network
}

\author{
Leila Fathi \\ Faculty of Computer Science and \\ Information Technology,Universiti \\ Putra Malaysia, 43400, Serdang, \\ Selangor, Malaysia \\ leila.fathi67@gmail.com
}

\author{
Hamidah Ibrahim \\ Faculty of Computer Science and \\ Information Technology, Universiti \\ Putra Malaysia, 43400, Serdang, \\ Selangor, Malaysia \\ hamidah@fsktm.upm.edu.my
}

\author{
Meghdad Mirabi \\ Faculty of Computer Science and \\ Information Technology,Universiti \\ Putra Malaysia, 43400, Serdang, \\ Selangor, Malaysia \\ meghdad.mirabi@gmail.com
}

\begin{abstract}
Recently, the use of XML for data broadcasting in mobile wireless networks has gained many attentions. One of the most essential requirements for such networks is data confidentiality. In order to secure XML data broadcast in mobile wireless network, mobile clients should obey a set of access authorizations specified on the original XML document. In such environments, mobile clients can only access authorized parts of encrypted XML stream based on their access authorizations. Several indexing methods have been proposed in order to have selective access to XML data over the XML stream. However, these indexing methods cannot be used for encrypted XML data. In this paper, we define a new unit structure for XML stream called SecNode which supports data confidentiality of XML data over the wireless broadcast channel. We also define two indexes for the SecNode structure called Min (NCS) and Min (NIS) to efficiently process XML queries over the encrypted XML stream. The experimental results demonstrate using two indexes Min (NCS) and Min (NIS) in the SecNode structure reduces the power consumption of mobile clients in processing XML queries over the encrypted XML stream.
\end{abstract}

\section{Categories and Subject Descriptors}

H.2.0 [DataBase Management]: General - Security, integrating, and protection; H.2.3 [DataBase Management]: Languages query languages; H.2.4 [DataBase Management]: Systems query processing, rule-based database, Textual database; H.3.3 [Information Storage and Retrieval]: Content and analysis and Indexing - Indexing Methods.

\section{General Terms}

Algorithms, Management, Measurement, Performance, Experimentation, Security, Standardization, Languages, Theory.

\section{Keywords}

Access Control, Data Confidentiality, Indexing, Mobile Wireless Broadcast, XML Query Processing, XML Stream.

\section{INTRODUCTION}

Recently, the use of XML (eXtensible Markup Language) [1] for data dissemination over wireless networks in many applications

Permission to make digital or hard copies of all or part of this work for personal or classroom use is granted without fee provided that copies are not made or distributed for profit or commercial advantage and that copies bear this notice and the full citation on the first page. To copy otherwise, or republish, to post on servers or to redistribute to lists, requires prior specific permission and/or a fee.

MobiDE '13, June 23, 2013 New York, New York, USA Copyright (C) 2013 ACM 978-1-4503-2197-6/13/06...\$15.00 like traffic and travel information systems has attracted many attentions.

In mobile wireless networks, access efficiency and energy consumption are two important issues as mobile clients carry hand held devices which are powered by limited battery and data processing capability $[4,5]$.

Access Time and Tuning Time [2-4] are two main performance metrics in a wireless broadcast channel. Access time measures the access efficiency in mobile wireless environments. Access time is the period of time which elapsed from the moment of submitting a query by a mobile client to the moment that it retrieves the desired data from the broadcast channel. Tuning time is the performance metric which estimates the energy consumption of mobile clients. Tuning time is the sum of the times that a mobile client elapses in the active mode to retrieve the desired data.

Data confidentiality is one of the most concerns in all public networks such as wireless broadcast networks. In order to support data confidentiality in such networks, data must be encrypted before sending over the broadcast channel.

Several indexing methods have been proposed in order to reduce tuning time such as those proposed by [4-7]. However, these indexing methods are only applicable for flat data and not for semi structured data such as XML because flat data is identified by keys while the XML data can be accessed by XML query languages like XPath [8] and XQuery [9].

Several indexing methods have been proposed to have selective access over an XML stream [10-12]. However, these indexing methods cannot be used for encrypted XML data since mobile clients can only access the authorized parts of encrypted XML stream.

Figure 1 shows an XML tree. Each XML node in Figure 1 is assigned with three rectangles where the left, middle, and right rectangles represent the accessibility of XML node to mobile clients of groups $g_{1}, g_{2}$ and $g_{3}$, respectively. In Figure 1, the gray rectangles represent accessibility of XML nodes to mobile clients of different groups while the white rectangles represent nonaccessibility of XML nodes to mobile clients of different groups. For example, the node "Border" is accessible to mobile clients of group $g_{2}$ but not to mobile clients of groups $g_{1}$ and $g_{3}$.

A broadcast stream of the XML tree illustrated in Figure 1 is shown in Figure 2. The broadcast server first encrypts all of the XML nodes separately with different keys and then organizes the encrypted XML nodes in the pre-order sequence and broadcasts them on the air. In Figure 2, each node is labeled by the Pre/Post labeling scheme proposed in [13]. The Pre/Post labeling scheme is able to determine all types of the structural relationships between 
arbitrary XML nodes which are needed when processing XML queries over the XML stream. An example of XML tree labeled by the Pre/Post labeling scheme is illustrated in Figure 1 where three values the preorder, postorder, and depth are assigned to each XML node in the XML tree.

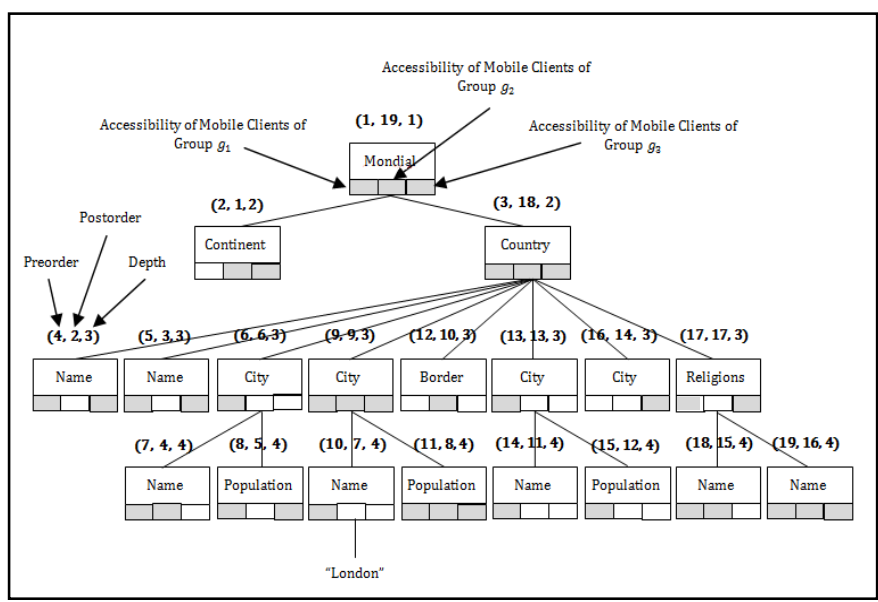

Figure 1. Accessibility of Different Mobile Clients over the XML Tree

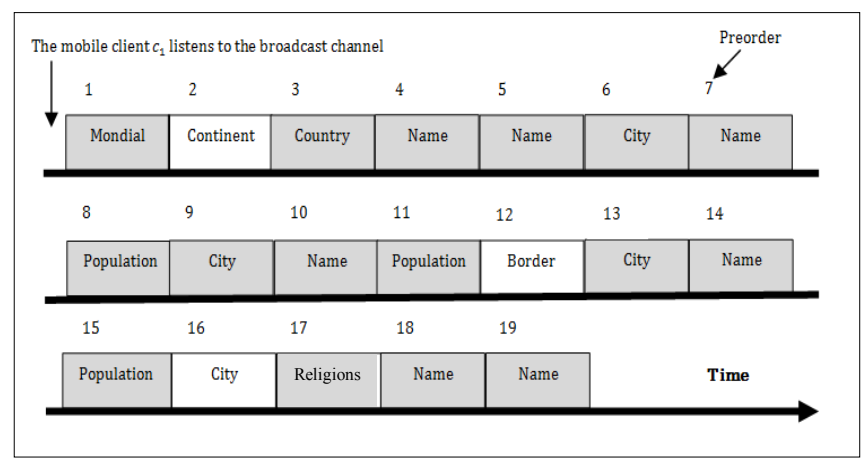

Figure 2. An Example of Encrypted XML Stream

In a conventional approach to process the XML query over an encrypted XML stream, first each node which is accessible by a mobile client should be decrypted using a specified key. Then, the mobile client should search for the appropriate nodes which can satisfy the XML query path. Assume that the mobile client $c_{1}$ which is a member of group $g_{1}$ listens to the broadcast channel before the root node is broadcasted and queries "/Mondial/ Country/Religions/Name". The mobile client $c_{1}$ can only decrypt the gray nodes in the stream shown in Figure 2 since it only has the set of keys for decrypting them based on the set of access authorizations specified for the members of group $g_{1}$ in the XML tree shown in Figure 1. In this case, the mobile client $c_{1}$ must decrypt 16 encrypted XML nodes (the number of gray nodes is 16) in the stream to find two candidate nodes "Name" (i.e. the nodes with the preorder 18 and 19). Such a process consumes a lot of energy to decrypt the XML nodes and find all of the candidate nodes satisfying the XML query "/Mondial/Country/Religions/ Name".

Motivated by the above example, this paper considers efficient ways to guarantee confidentiality of XML data in the stream and selectively access over the XML stream in a wireless broadcast channel. Hence, the main contributions of this paper are summarized as follows:

- A unit structure of XML stream called SecNode is defined in order to provide confidentiality of XML data in wireless stream. The SecNode structure consists of XML data and index information. The field Root-to-Node Path in the SecNode is defined to identify the exact path of SecNode in the stream in processing XML queries.

- We define two indexes Min (NCS) and Min (NIS) for the SecNode structure based on the set of access authorizations specified in the original XML document in order to selectively access over the encrypted XML stream. Min (NCS) points to the next nearest accessible XML node which can satisfy the XML query while Min (NIS) points to the next irrelevant accessible node which is not a query answer.

- We propose algorithms to generate an encrypted XML stream based on the SecNode structure and process simple path XML queries over the encrypted XML stream.

The remainder of this paper is organized as follows: In Section 2, the unit structure of XML stream called SecNode is defined. In Section 3, the process of generating an encrypted XML stream from the original XML document based on the SecNode structure is explained. In Section 4, the process of XML querying over the encrypted XML stream is described. In Section 5, the experimental results in processing XML queries over the encrypted XML stream are presented. Finally, in Section 6, the paper is concluded with a conclusion and discussion of future works.

\section{UNIT STRUCTURE OF XML STREAM}

In order to securely broadcast the XML data, first an XML stream should be generated. For generating XML stream in a secure manner, XML stream should be organized in a suitable representation which unauthorized mobile clients cannot have access to data. To guarantee the confidentiality of data, the organized XML stream should be encrypted before broadcasting over the channel. For supporting XML query processing at mobile clients, some index information should be applied to the generated XML stream which are based on access authorizations specified in the original XML document.

Figure 3 (a) shows the structure of SecNode for node $e$ $\left(\mathrm{SecNode}_{e}\right.$ ) in the stream which contains the following fields: Key ID, Preorder, Postorder, Root-to-Node Path, Minimal of Next Candidate nodes Set (Min (NCS)), Minimal of Next Irrelevant nodes Set (Min (NIS)), Text, and Attributes List (AL) of node $e$.

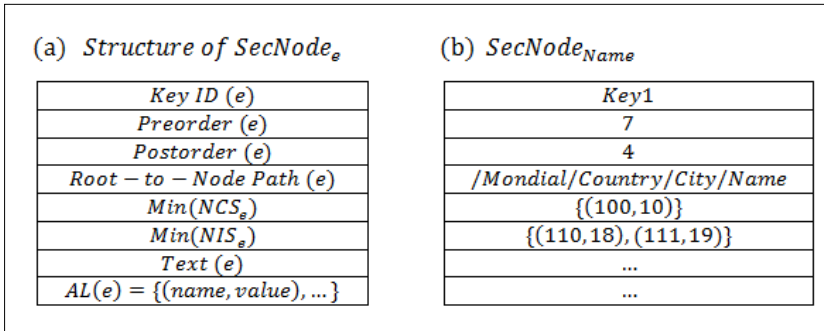

Figure 3. Structure of SecNode

The field Key ID $(e)$ in SecNode ${ }_{e}$ is the identifier of key used for encryption/decryption of $\operatorname{SecNode}_{e}$. The fields Preorder $(e)$ and Postorder (e) in $\operatorname{SecNode}_{e}$ are the preorder and postorder of node $e$ 
when the XML tree $T$ is traversed in the preorder and postorder sequence, respectively. The fields Text $(e)$ and $\mathrm{AL}(e)$ in SecNode $_{e}$ represent the content and list of attributes of node $e$ in the XML tree $T$, respectively. The field AL $(e)$ contains a set of pairs (name, value) representing the name and value of each attribute of node $e$.

Definition 1: The Root-to-Node Path of node $e$ in the XML tree $T$ is a sequence of node names (or tag names) from the root node $r$ to node $e$ which are separated by "/".

For example, the Root-to-Node Path of node "Population" with the preorder 15 (called node 15) in the XML tree illustrated in Figure 1 is the path "/Mondial/Country/City/Population".

The field Root-to-Node Path (e) in SecNode ${ }_{e}$ is used by mobile clients to identify the exact path of SecNode in the stream in processing XML queries.

Definition 2: Node $f$ is the next candidate node of node $e$ in the XML tree $T$ if it satisfies the following two conditions:

- $\quad$ Node $f$ is after node $e$ when the XML tree $T$ is traversed in preorder;

- $\quad$ Node $f$ has the same Root-to-Node Path as node $e$.

For example, node 9 (i.e. node "City") with the Root-to-Node Path "/Mondial/Country/City" is the next candidate node of node 6 (i.e. node "City") with the Root-to-Node Path "/Mondial/Country/ City" in the XML tree illustrated in Figure 1.

Definition 3: Let $U=\left\{u_{1}, u_{2}, \ldots, u_{n}\right\}$ be the set of users (or mobile clients) and $U G=\left\{g_{1}, g_{2}, \ldots, g_{m}\right\}$ be the set of user groups. We suppose that $M: U \rightarrow U G$ be a function that maps a user (or mobile client) in $U$ to a user group in $U G$. The authorization code of node $e$ in the XML tree $T$ is defined by $A C_{e}=a_{1} a_{2} \ldots a_{m}$ where

$\forall 1 \leq i \leq m, a_{i}= \begin{cases}1, & \text { if the ith user group in } U G, g_{i}, \text { has access to node } e \\ 0, & \text { otherwise }\end{cases}$

and $m$ is the total number of user groups in UG.

For example, node 12 (i.e. node "Border") in the XML tree illustrated in Figure 1 can only be accessed by mobile clients which are members of group $g_{2}$. Therefore, the authorization code of node 12 is $010\left(A C_{\text {node } 12}=010\right)$.

Definition 4: Let node $f$ be the next candidate node of node $e$, $A C_{f}$ be the authorization code of node $f$, and $A d d_{f}$ be the address (arrival time) of node $f$ in the stream. The set of next candidate nodes of node $e\left(N C S_{e}\right)$ is an ordered set of the next candidate nodes of node $e$ defined as follows:

$$
N C S_{e}=\left\{\left(A C_{f}, A d d_{f}\right) \mid A C_{f} \neq 0^{m}(m \text { occurrences of } 0)\right\}
$$

where $m$ is the total number of user groups in $U G$ and the pairs of $\left(A C_{f}, A d d_{f}\right)$ are ordered in ascending based on their address (Add).
For example, the set of next candidate nodes of node 7 (i.e. node "Name") in the XML tree illustrated in Figure 1 is as follows: $N C S_{\text {node } 7}=\{(100,10),(100,14)\}$.

To obtain the minimal of next candidate nodes set of each XML node in the XML tree $T$, we define a function called FindMinimalofNextCandidateSet as illustrated in Figure 4.

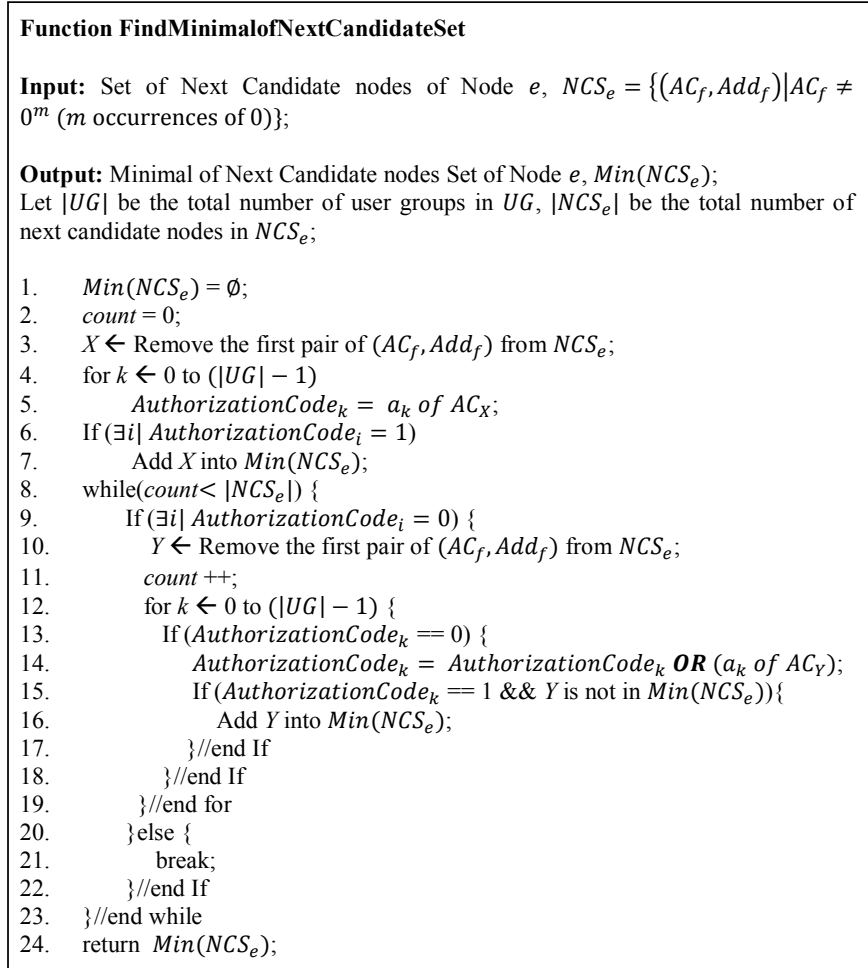

Figure 4. FindMinimalofNextCandidateSet

The field Min (NCS) in SecNode is a pointer to the next accessible candidate node in the XML stream based on the access authorizations specified in the XML tree $T$. At the processing time of an XML query, mobile clients use this field to jump forward to the next accessible XML node which is a query answer. It should be noted that the next candidate nodes may be inaccessible to the mobile client which queries from the stream.

Given the set of next candidate nodes of node $e\left(N C S_{e}\right)$, the function FindMinimalofNextCandidateSet removes the first next candidate node from $N C S_{e}$ and stores it as $X$ (Line 3). It also stores the authorization code of $X\left(A C_{X}\right)$ as AuthorizationCode (Lines 4-5). The function adds $X$ into the Minimal of Next Candidate nodes Set $\left(\operatorname{Min}\left(N C S_{e}\right)\right)$ as the first next candidate node if at least one bit of AuthorizationCode (e.g. the $k$ th bit) is " 1 " (Lines 6-7) since $X$ is at least accessible to a user group (e.g. the $k$ th user group) and therefore, it is the next accessible candidate node of that user group. In the case that all bits of AuthorizationCode are " 0 ", the first candidate node (i.e. $X$ ) is not accessible to all user groups and therefore, it cannot be a member of Minimal of Next Candidate nodes Set of node $e$ $\left(\operatorname{Min}\left(N C S_{e}\right)\right)$. In the case that all the bits of AuthorizationCode are "1", $\operatorname{Min}\left(N C S_{e}\right)$ only contains one next candidate node (i.e. $X)$ since this node is accessible to all user groups. In the case that at least one bit of AuthorizationCode is "0" (Line 9), the function removes the first next candidate node from $N C S_{e}$ and stores it as $Y$ (Line 10). It then computes the bitwise operation $O R$ 
over the $k$ th bit of AuthorizationCode and the $k$ th bit of $A C_{Y}$ if the $k$ th bit of AuthorizationCode is " 0 " (Lines 13-14). In the case that the result of bitwise operation $O R$ is "1", the next candidate node (i.e. $Y$ ) is added to $\operatorname{Min}\left(N C S_{e}\right)$ (Lines 15-16) since this candidate node (i.e. $Y$ ) is accessible to the $k$ th user group and is the next accessible candidate node of that group. This process continues until all of the next candidate nodes of $N C S_{e}$ are processed or all of the bits of AuthorizationCode are "1".

For example, the set of next candidate nodes of node 6 (i.e. node "City") in the XML tree illustrated in Figure 1 is $N C S_{\text {node } 6}=$ $\{(111,9),(100,13),(001,16)\}$ and the Minimal of Next Candidate nodes Set of node 6 is $\operatorname{Min}\left(N C S_{\text {node } 6}\right)=\{(111,9)\}$ since node 9 (i.e. node "City") is accessible to all user groups $\left(A C_{\text {node } 9}=111\right)$.

Definition 5: Node $f$ is the next irrelevant node of node $e$ in the XML tree $T$ if it satisfies the following three conditions:

- $\quad$ Node $f$ is after node $e$ when the XML tree $T$ is traversed in preorder;

- $\quad$ Node $f$ has the same depth as node $e$;

- The Root-to-Node Path of parent node of node $f$ is different from that of node $e$.

For example, node 18 (i.e. node "Name") is the next irrelevant node of node 7 (i.e. node "Name") in the XML tree illustrated in Figure 1 since the Root-to-Node Path of parent node of node 7 (i.e. "/Mondial/Country/City") is different from that of node 18 (i.e. "/Mondial/Country/Religions").

Definition 6: Let node $f$ be the next irrelevant node of node $e$, $A C_{f}$ be the authorization code of node $f$, and $A d d_{f}$ be the address (arrival time) of node $f$ in the stream. The set of next irrelevant nodes of node $e\left(N I S_{e}\right)$ is an ordered set of the next irrelevant nodes of node $e$ defined as follows:

$$
N I S_{e}=\left\{\left(A C_{f}, A d d_{f}\right) \mid A C_{f} \neq 0^{m}(m \text { occurrences of } 0)\right\}
$$

where $m$ is the total number of user groups in $U G$ and the pairs of $\left(A C_{f}, A d d_{f}\right)$ are ordered in ascending based on their address (Add).

For example, the set of next irrelevant nodes of node 7 (i.e. node "Name") in the XML tree illustrated in Figure 1 is as follows: $N I S_{\text {node } 7}=\{(110,18),(111,19)\}$.

In order to obtain the minimal of next irrelevant nodes set of each XML node in the XML tree $T$, we define a function called FindMinimalofNextIrrelevantSet as illustrated in Figure 5.

The field Min (NIS) in SecNode is a pointer to the next accessible irrelevant node in the XML stream based on the access authorizations specified in the XML tree $T$. At the processing time of an XML query, mobile clients use this field to skip the irrelevant accessible nodes of XML query. It should be noted that the next irrelevant nodes may be inaccessible to the mobile client which queries from the stream.

Given the set of next irrelevant nodes of node $e\left(N I S_{e}\right)$, the function FindMinimalofNextIrrelevantSet removes the first next irrelevant node from $N I S_{e}$ and stores it as $X$ (Line 3). It also stores the authorization code of $X\left(A C_{X}\right)$ as AuthorizationCode
(Lines 4-5). The function adds $X$ into the Minimal of Next Irrelevant nodes Set $\left(\operatorname{Min}\left(N I S_{e}\right)\right)$ as the first next irrelevant node if at least one bit of AuthorizationCode (e.g. the $k$ th bit) is " 1 " (Lines 6-7) since $X$ is at least accessible to a user group (e.g. the $k$ th user group) and therefore, it is the next accessible irrelevant node of that user group. In the case that all bits of AuthorizationCode are "0", the first next irrelevant node (i.e. $X$ ) is not accessible to all user groups and therefore, it cannot be a member of Minimal of Next Irrelevant nodes Set of node $e$ $\left(\operatorname{Min}\left(N I S_{e}\right)\right)$. In the case that all the bits of AuthorizationCode are "1", $\operatorname{Min}\left(N I S_{e}\right)$ only contains one next irrelevant node (i.e. $\left.X\right)$ since this node is accessible to all user groups. In the case that at least one bit of AuthorizationCode is " 0 " (Line 9), the function removes the first next irrelevant node from $N I S_{e}$ and stores it as $Y$ (Line 10). It then computes the bitwise operation $O R$ over the $k$ th bit of AuthorizationCode and the $k$ th bit of $A C_{Y}$ if the $k$ th bit of AuthorizationCode is " 0 " (Lines 13-14). In the case that the result of bitwise operation $O R$ is " 1 ", the next irrelevant node (i.e. $Y$ ) is added to $\operatorname{Min}\left(N I S_{e}\right.$ ) (Lines 15-16) since this irrelevant node (i.e. $Y$ ) is accessible to the $k$ th user group and is the next irrelevant node of that group. This process continues until all of the next irrelevant nodes of $N I S_{e}$ are processed or all of the bits of AuthorizationCode are "1".

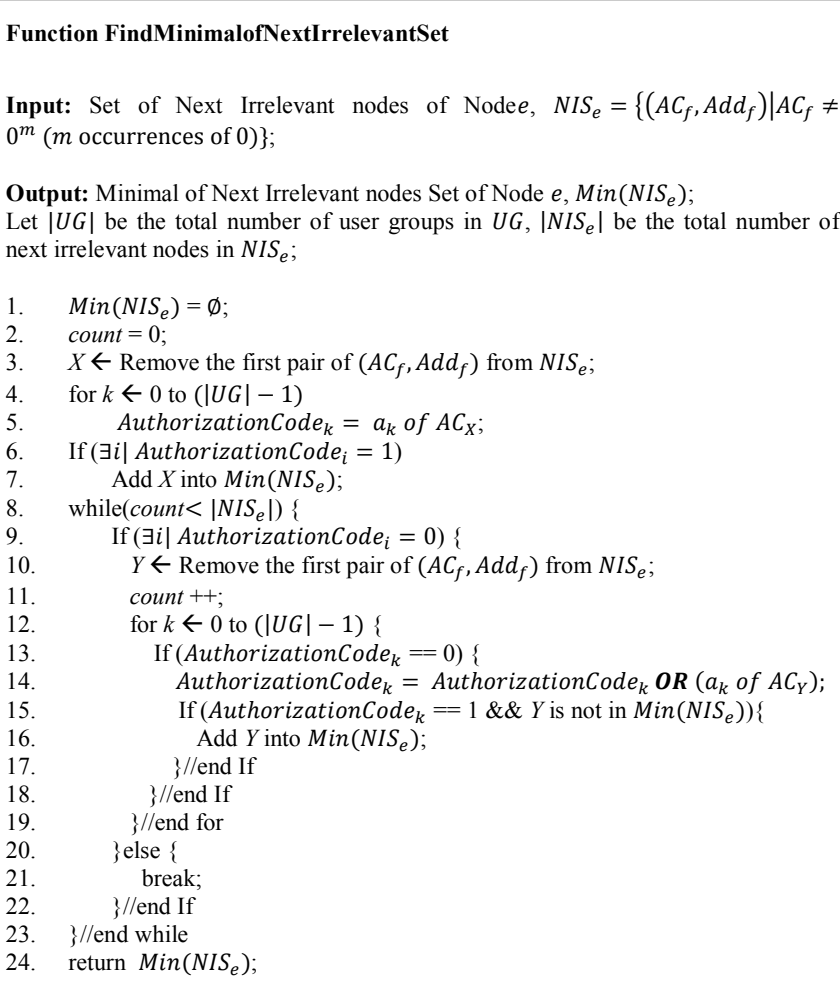

Figure 5. FindMinimalofNextIrrelevantSet

\section{PROCESS OF GENERATING AN ENCRYPTED XML STREAM}

The EncryptedXMLStreamGenerator algorithm is illustrated in Figure 6. The EncryptedXMLStreamGenerator algorithm generates an encrypted XML stream based on SecNode structure. In order to generate an encrypted XML stream, we use the SAX API which is an event-driven API for parsing XML documents. 


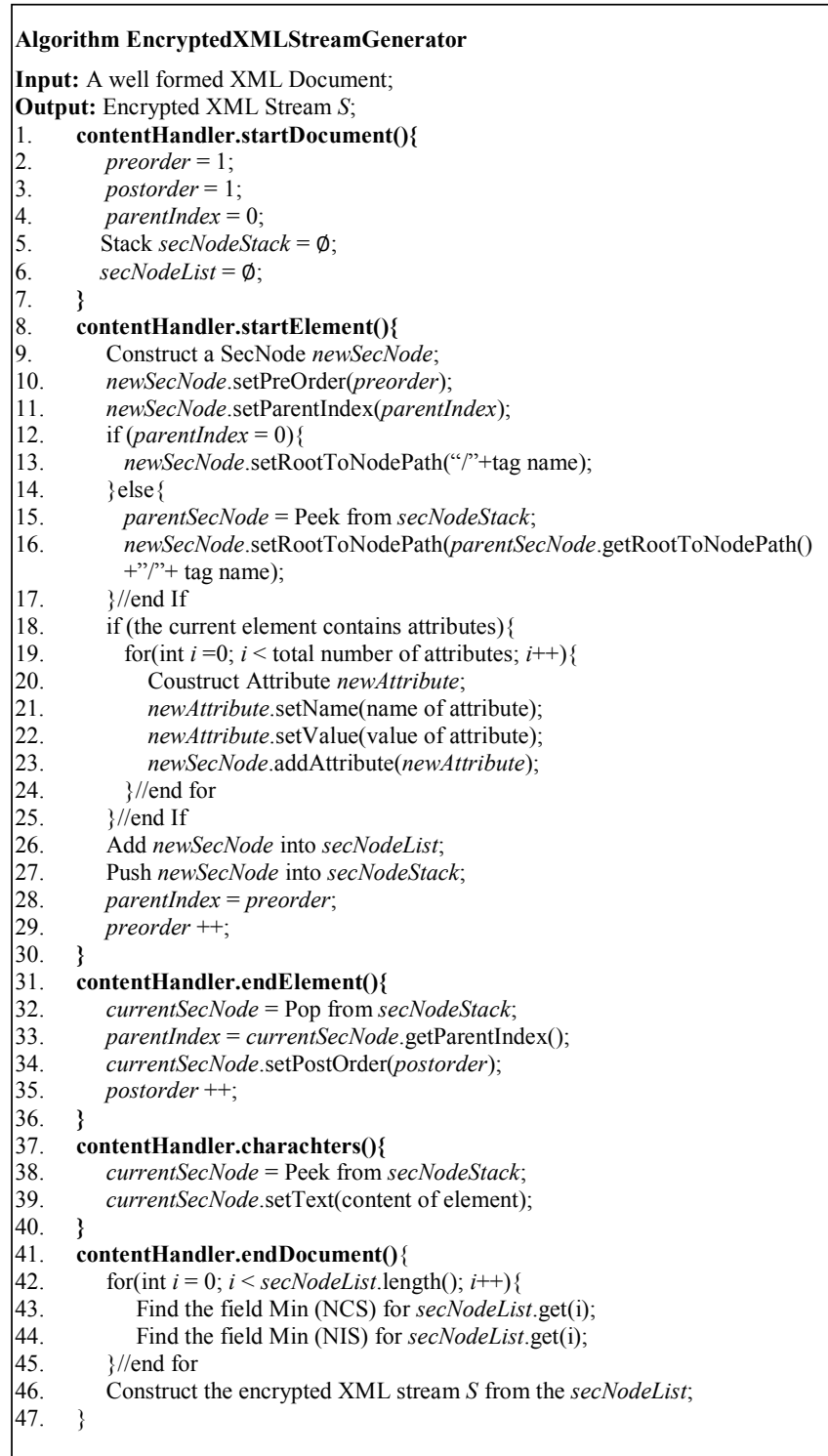

Figure 6. EncryptedXMLStreamGenerator Algorithm

In this algorithm, the encrypted XML stream generator first initializes related parameters by executing the startDocument() event handler (Lines 1-7). When a start XML tag is encountered during parsing of an XML document, the encrypted XML stream generator creates a new SecNode namely newSecNode (Line 9) and sets its related parameters (Lines 10-25) by executing the startElement() event handler. It also adds newSecNode into the list of SecNodes (secNodeList) (Line 26). Since some parameters of newSecNode are not set, the encrypted XML stream generator pushes newSecNode into a stack (secNodeStack) (Line 27). In the case that the current XML element contains text content, the encrypted XML stream generator sets its related parameters by executing the characters() event handler (Lines 37-40). When an end XML tag is encountered during parsing of an XML document, the encrypted XML stream generator executes the endElement() event handler to set some parameters of newSecNode such as the postorder (Lines 31-36). When the whole of XML document is parsed, the encrypted XML stream generator executes the
endDocument() event handler and constructs the encrypted XML stream for the parsed XML document by exploiting the set of SecNodes in the secNodeList (Lines 41-47). It first finds the two fields Min(NCS) and Min(NIS) for the set of SecNodes (Lines 42-45) and then constructs the encrypted XML stream from the set of SecNodes in the secNodeList (Line 46). Note that each SecNode is encrypted with a unique key.

\section{XML QUERY PROCESSING OVER THE ENCRYPTED XML STREAM}

In this section, we explain how a mobile client can process different types of XML queries over an encrypted XML stream using the SecNode structure.

To process simple path XML queries over the encrypted XML stream, algorithm SimplePathQueryProcessing is defined as illustrated in Figure 7.

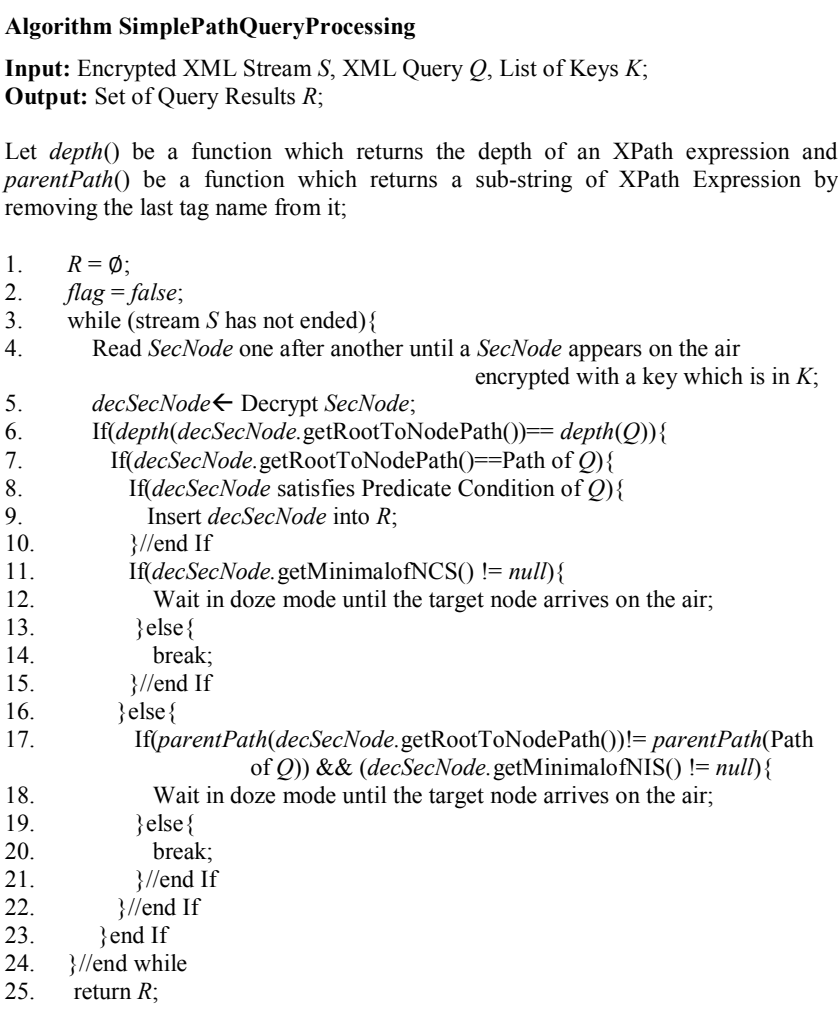

Figure 7. SimplePathQueryProcessing Algorithm

Given an XML query $Q$, a mobile client listens to the broadcast channel and reads SecNode on the air one after another until a SecNode encrypted with the mobile client's key appears in the stream (Line 4). Then, the mobile client decrypts the SecNode and stores it as decSecNode(Line 5). It also compares the depth of Root - to - Node Path of decSecNode with the depth of XML query path (Line 6). In the case that the depth of Root to - Node Path of decSecNode is equal to that of XML query path, the mobile client compares the Root - to - Node Path of decSecNode with the XML query path (Line 7) and adds the decSecNode to the set $R$ if they match. If the XML query $Q$ contains predicate conditions over the text content or attributes, the mobile client also investigates whether the decSecNode 
satisfies the predicate conditions or not (Lines 8-10). After finding the first accessible candidate node satisfying the XML query $Q$ (i.e. decSecNode), the mobile client jumps forward to the next accessible SecNode having the same Root - to - Node Path by following the field $\operatorname{Min}(N C S)$ contained in the decSecNode (Lines 11-15). In the case that the decSecNode and the XML query $Q$ have the same depth but different Root-toNode Path, none of the accessible SecNodes having the same Root - to - Node Path as the decSecNode satisfy the XML query $Q$ and then, the mobile client jumps forward to the next accessible SecNode having the same depth but a different Root - to - Node Path by using the field $\operatorname{Min}(N I S)$ in the decSecNode (Lines 17-21).

An example of simple path query processing over the encrypted XML stream using the field $\operatorname{Min}(N C S)$ is illustrated in Example 1.

Example 1: As shown in Figure 8, assume that the mobile client $c_{1}$ which is a member of group $g_{1}$ listens to the broadcast channel when node 3 (i.e. node "Country") is on the air and submits the XML query "/Mondial/Country/City/Population".

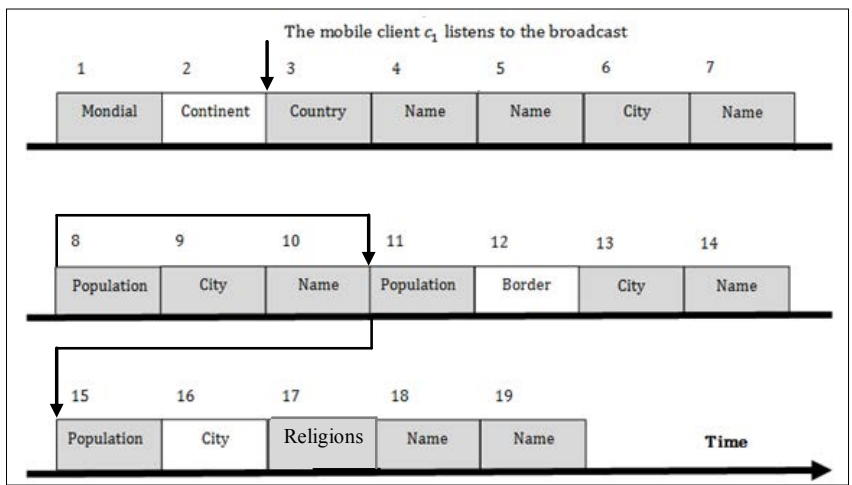

Figure 8. An Example of Simple Path Query Processing using the Field Min (NCS)

The mobile client $c_{1}$ can decrypt node 3 since the Key $I D$ of node 3 matches with one of the Key IDs of the mobile client $c_{1}$. After decrypting node 3 , the mobile client $c_{1}$ computes the depth of node 3 using the field Root - to - Node Path of node 3. Since the depth of node 3 is different from that of the submitted XML query, the mobile client $c_{1}$ continues to read the encrypted SecNodes in the stream. After reading and decrypting node 8 , the mobile client $c_{1}$ compares the field Root - to - Node Path of node 8 with the submitted XML query path. The mobile client $c_{1}$ finds that node 8 satisfies the XML query and therefore, it adds node 8 to the set $R$. By exploiting the field $\operatorname{Min}(N C S)$ of node 8 (i.e. $\operatorname{Min}\left(N C S_{\text {node } 8}=\{(111,11)\}\right)$, the mobile client $c_{1}$ can predict arrival time of the next accessible candidate node (i.e. node 11) on the air. Therefore, the mobile client $c_{1}$ switches to the doze mode to conserve its battery power. When node 11 is on the air, the mobile client $c_{1}$ wakes up and reads node 11 . In the same manner, the mobile client $c_{1}$ uses the field $\operatorname{Min}(N C S)$ of node 11 to access the next accessible candidate node of node 11 (i.e. node $15)$.

An example of processing simple path XML queries over the encrypted XML stream using the field $\operatorname{Min}(N I S)$ is illustrated in Example 2.
Example 2: As shown in Figure 9, assume that the mobile client $c_{2}$ which is a member of group $g_{2}$ listens to the broadcast channel when node 5 is on the air and submits the XML query "/Mondial/Country/Religions /Name".

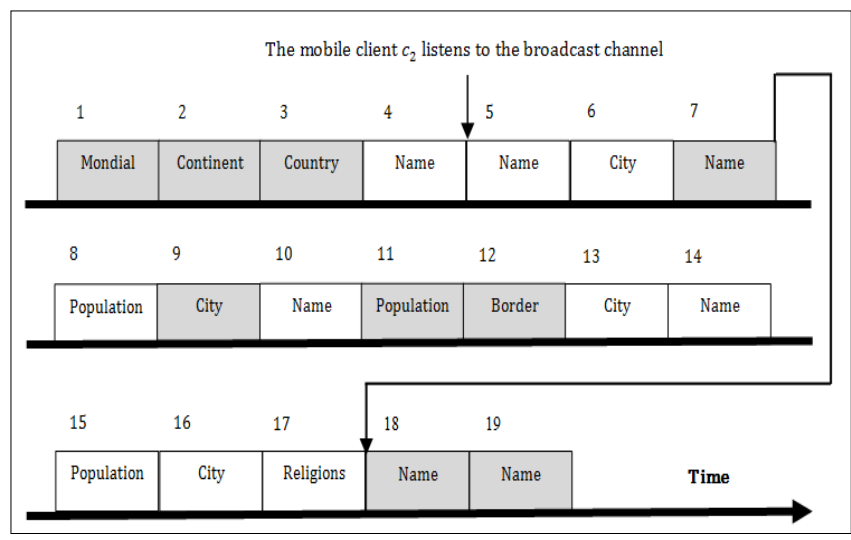

Figure 9. An Example of Simple Path Query Processing using the Field Min (NIS)

The mobile client $c_{2}$ cannot decrypt node 5 since the Key ID of node 5 does not match with any of the Key IDs of the mobile client $c_{2}$. Therefore, the mobile client $c_{2}$ continues to read from the broadcast channel. When node 7 is on the air, the mobile client $c_{2}$ decrypts it since the Key ID of node 7 matches with one of the Key IDs of the mobile client $c_{2}$. After decrypting node 7 , the mobile client $c_{2}$ computes the depth of node 7 using the field Root - to - Node Path of node 7. The mobile client $c_{2}$ finds that the depth of node 7 is equal to the depth of the given XML query but the Root - to - Node Path of node 7 is different from the path of XML query. Since none of the accessible nodes having the same Root - to - Node Path as node 7 can be the result of the XML query, the mobile client $c_{2}$ can efficiently skip the irrelevant accessible nodes in the stream by using the field $\operatorname{Min}(N I S)$ of node 7 (i.e. $\operatorname{Min}\left(N I S_{\text {node } 7}=\right.$ $\{(110,18),(111,19)\}))$. By exploiting the field Min(NIS) of node 7 , the mobile client $c_{2}$ can predict the arrival time of node 18 on the air. Therefore, the mobile client $c_{2}$ switches to the doze mode to conserve its battery power. When node 18 is on the air, the mobile client $c_{2}$ wakes up and reads node 18 . The mobile client $c_{2}$ finds the first answer at node 18 since it has the same Root to - Node Path as the XML query.

\section{PERFORMANCE EVALUATION}

In this section, we evaluate the performance of our proposed indexing method for the SecNode structure in processing XML queries by performing several experiments.

All the experiments were conducted on a system with the Intel Pentium(R) $2.20 \mathrm{GHz}$ processor and 2GB RAM running Windows 7 Ultimate where the server and client modules were implemented in Java.

\subsection{Experimental Setting}

We logically modeled the encrypted XML stream as a binary file, where the broadcast server writes a byte stream on the file and the mobile clients read the file and process XML queries. We used the algorithm 3-DES for encryption/decryption of SecNodes in the file. 
In our simulation model, we assumed that the broadcast bandwidth is fully utilized for broadcasting the encrypted XML stream. To measure the tuning time, we considered only the activity of a mobile client since the activity of a mobile client does not effect on the performance of XML querying at other mobile clients.

We assumed that the encrypted XML stream is broadcasted and accessed in units with a fixed size (i.e. buckets) and thus we measured the tuning time in processing XML queries by the number of buckets. The bucket is the smallest logical unit in a wireless broadcast channel. In the view of assumption that the network speed is fixed, the number of buckets can be converted into the time since the elapsed time for reading a bucket is computed as the bucket size divided by the network speed [4].

To measure the performance variation based on the number of buckets, we used three different bucket sizes, 64, 128, and 256 bytes in our experiments. However, we only present the experimental results for the cases that the bucket size is set to 128 bytes since the experimental results do not dependent on the bucket size.

To measure the performance variation based on the types of XML data sets, we used two data sets XMark and Shakespeare. The XMark data set as a syntactic XML data set was generated by the XML document generator xmlgen proposed by [14] while the Shakespeare data set was collected from the XML data repository at the University of Washington ${ }^{1}$. Table 1 shows the characteristics of two XML data sets used in our experiments.

Table 1. XML Data Sets

\begin{tabular}{|c|c|c|c|c|c|}
\hline Data Set & $\begin{array}{c}\text { Size } \\
(\mathrm{KB})\end{array}$ & $\begin{array}{c}\text { Number of } \\
\text { Elements }\end{array}$ & $\begin{array}{c}\text { Number of } \\
\text { Attributes }\end{array}$ & $\begin{array}{c}\text { Max } \\
\text { Depth }\end{array}$ & $\begin{array}{c}\text { Max Fan } \\
\text { Out }\end{array}$ \\
\hline XMark & 1155 & 17132 & 3919 & 12 & 255 \\
\hline Shakespeare & 1061 & 25339 & 0 & 7 & 162 \\
\hline
\end{tabular}

To measure the performance variation based on the types of XML queries, we used different types of XPath queries. The list of XPath queries used in our experiments is shown in Table 2.

Table 2. XPath Query Sets of the Different XML Data Sets

\begin{tabular}{|c|c|c|}
\hline $\begin{array}{c}\text { Data } \\
\text { Set }\end{array}$ & $\begin{array}{l}\text { Query } \\
\text { Name }\end{array}$ & XPath Expression \\
\hline \multirow{5}{*}{ XMark } & XM1 & site/regions/samerica/item \\
\hline & XM2 & site/categories/category/description/parlist \\
\hline & XM3 & site/open_auctions/open_auction/bidder/date \\
\hline & XM4 & site/people/person/address/country[text()="United States"] \\
\hline & XM5 & site/people/person/profile[@income="9876.00] \\
\hline \multirow{5}{*}{ Sheakspeare } & SH1 & /PLAYS/PLAY/ACT \\
\hline & SH2 & /PLAYS/PLAY/PERSONAE/PGROUP \\
\hline & $\mathrm{SH} 3$ & /PLAYS/PLAY/ACT/EPILOGUE/SPEECH/SPEAKER \\
\hline & SH4 & $\begin{array}{l}\text { PLAYS/PLAY/ACT/EPILOGUE/TITLE[text()="EPILOG } \\
\text { UE"] }\end{array}$ \\
\hline & SH5 & $\begin{array}{l}\text { /PLAYS/PLAY/ACT/SCENE/SPEECH/STAGEDIR[@SPE } \\
\text { AKER="BERTRAM"] }\end{array}$ \\
\hline
\end{tabular}

$1_{\text {http://www.cs.washington.edu/research/xmldatasets }}$
The XPath queries with the query types 1,2 , and 3 were used to test the simple path XML queries. To measure the performance variation based on the depth of XML queries, we used the simple path XML queries with different depths (See the XPath queries with the query types 1,2 , and 3 in Table 2). The XPath queries with the query type 4 were used to test the XML queries having a predicate condition on the text content. The XPath queries with the query type 5 were used to test the XML queries having a predicate condition on the attribute value.

In our simulation model, we randomly and uniformly selected the set of XML nodes from the XML data sets as the accessible nodes. We controlled the accessibility of the XML nodes with the accessibility ratio. The accessibility ratio is the fraction of the XML nodes in the XML data set which are accessible. To measure the performance variation based on the accessibility ratio of the XML nodes, we varied the accessibility ratio from $10 \%$ to $90 \%$ with an interval $20 \%$.

The performance metric used in our experiment was the Tuning Time Ratio. It is defined as follows:

Tuning Time Ratio $=(($ Number of buckets to Read in Encrypted XML Stream which uses the SecNode structure) / (Number of Buckets to Read in Encrypted XML Stream which does not use the SecNode Structure) $) * 100$

\subsection{Experimental Results on Tuning Time}

Figure 10 and Figure 11 show the Tuning Time Ratio in processing different types of XML queries when the accessibility ratio is varied $(10 \% \sim 90 \%)$ on the XMark data set and the Shakespeare data set, respectively.

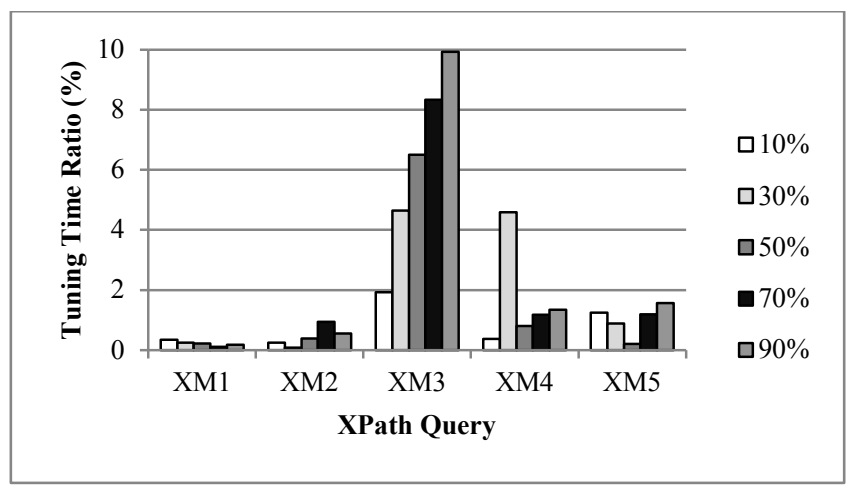

Figure 10. Tuning Time Ratio on the XMark Data Set

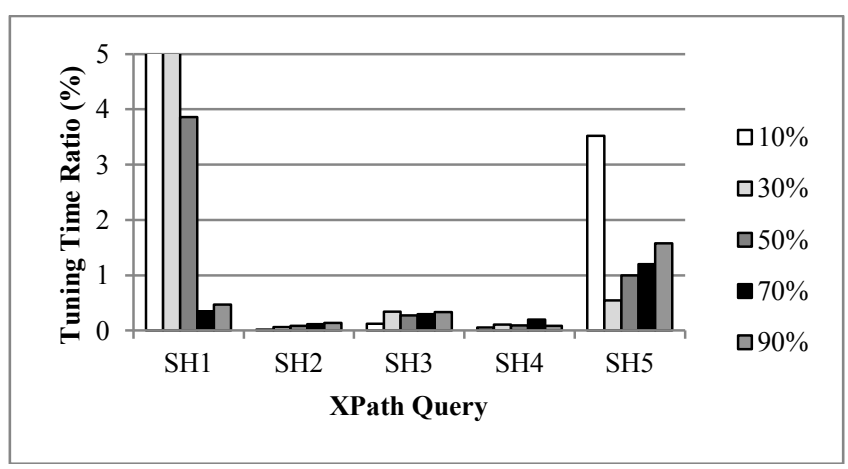

Figure 11. Tuning Time Ratio on the Shakespeare Data Set 
From Figure 10 and Figure 11, it is clear that the Tuning Time Ratio changes according to the properties of XPath queries and XML data sets. The average Tuning Time Ratios on the XMark data set and the Shakespeare data set are $1.91 \%$ and $1.64 \%$, respectively. It means that the use of two indexes Min (NCS) and Min (NIS) in the SecNode structure reduces the power consumption of mobile clients in processing XML queries over the encrypted XML stream.

\section{CONCLUSION AND FUTURE WORKS}

In this paper, we defined a unit structure of XML stream called SecNode which provides confidentiality of XML nodes in the stream. The SecNode structure contains the field Root-to-Node Path to process the simple path XML queries. Two indexes Min (NCS) and Min (NIS) were defined for the SecNode structure to reduce the tuning time in processing XML queries.

By performing several experiments on the different XML data sets, we demonstrated that the use of SecNode structure for secure streaming the XML nodes in a wireless broadcast channel reduces the power consumption of mobile clients in processing XML queries over the encrypted XML stream.

In the future, we intend to investigate other issues which are not considered in this paper. First, we attempt to extend our work to support twig pattern XML queries having several wildcards, descendant axes, and predicate conditions at the different steps of the XPath expressions. Second, one of the most important issues in error-prone environments such as a wireless broadcast channel is robustness. The reason is that the access sequence induced by the index information should be followed exactly at the query processing time. Otherwise, the whole of query processing may fail. Therefore, we attempt to extend our work to be used in an error-prone mobile wireless network.

\section{REFERENCES}

[1] Bray, T., Paoli, J., Sperberg-McQueen, C.M., Maler, E., and Yergeau, F. 2008. Extensible Markup Language (XML) 1.0 (5th Edition) W3C Recommendation. http://www.w3.org/TR/REC-xml/.

[2] Imieĺinski, T. and Badrinath, B.R. 1993. Data Management for Mobile Computing. SIGMOD Record. 22, 1, 34-39.

[3] Acharya, S., Alonso, R., Franklin, M., and Zdonik, S. 1995. Broadcast Disks: Data Management for Asymmetric Communication Environments. In Proceedings of the 1995 ACM SIGMOD International Conference on Management of Data. ACM, San Jose, California, USA, 199-210.

[4] Imielinski, T., Viswanathan, S., and Badrinath, B.R. 1997. Data on air: Organization and Access. IEEE Transactions on Knowledge and Data Engineering. 9, 3, 353-372.

[5] Chung, Y.D., Yoo, S., and Kim, M.H. 2010. Energy and Latency Efficient Processing of Full-Text Searches on a Wireless Broadcast Stream. IEEE Transactions on Knowledge and Data Engineering. 22, 2, 207-218.

[6] Chen, M.-S., Wu, K.-L., and Yu, P.S. 2003. Optimizing Index Allocation for Sequential Data Broadcasting in
Wireless Mobile Computing. IEEE Transactions on Knowledge and Data Engineering. 15, 1, 161-173.

[7] Chung, Y.D. and Kim, M.H. 2000. An Index Replication Scheme for Wireless Data Broadcasting. Journal of Systems and Software. 51, 3, 191-199.

[8] Berglund, A., Boag, S., Chamberlin, D., Fernández, M.F., Kay, M., Robie, J., and Siméon, J. 2010. XML Path Language (XPath) $2.0 \quad$ (Second Edition) http://www.w3.org/TR/xpath20/.

[9] Boag, S., Chamberlin, D., Fernández, M.F., Florescu, D., Robie, J., and Siméon, J. 2010. XQuery 1.0: An XML Query Language (Second Edition). http://www.w3.org/TR/xquery/.

[10] Park, C.-S., Kim, C.S., and Chung, Y.D. 2005. Efficient Stream Organization for Wireless Broadcast of XML Data. In Proceedings of the 10th Asian Computing Science Conference on Advance in Computer Science. Springer, Kunming, China, 223-235.

[11] Park, J.P., Park, C.-S., and Chung, Y.D. 2010. Energy and Latency Efficient Access of Wireless XML Stream. Journal of Database Management. 21, 1, 58-79.

[12] Park, S.-H., Choi, J.-H., and Lee, S. 2006. An Effective, Efficient XML Data Broadcasting Method in a Mobile Wireless Network. In Proceedings of the 17th International Conference on Dtabase and Expert Systems Applications (DEXA 2006). Springer, Krakow, Poland, 358-367.

[13] Dietz, P.F. 1982. Maintaining Order in a Linked List. In Proceedings of the fourteenth Annual ACM Symposium on Theory of Computing. ACM, San Francisco, California, USA, 122-127.

[14] Schmidt, A., Waas, F., Kersten, M., Carey, M.J., Manolescu, I., and Busse, R. 2002. XMark: A Benchmark for XML Data Management. In Proceedings of the 28th International Conference on Very Large Data Base. Hong Kong, China, 974-985. 